\title{
On Multipath Fading Channels at High SNR
}

\author{
Tobias Koch Amos Lapidoth \\ ETH Zurich, Switzerland \\ Email: \{tkoch, lapidoth\}@isi.ee.ethz.ch
}

\begin{abstract}
This paper studies the capacity of discrete-time multipath fading channels. It is assumed that the number of paths is finite, i.e., that the channel output is influenced by the present and by the $L$ previous channel inputs. A noncoherent channel model is considered where neither transmitter nor receiver are cognizant of the fading's realization, but both are aware of its statistic. The focus is on capacity at high signal-to-noise ratios (SNR). In particular, the capacity pre-loglog-defined as the limiting ratio of the capacity to $\log \log (\mathrm{SNR})$ as SNR tends to infinity-is studied. It is shown that, irrespective of the number of paths $L$, the capacity pre-loglog is 1 .
\end{abstract}

\section{INTRODUCTION}

This paper studies the capacity of multipath (frequencyselective) fading channels. A noncoherent channel model is considered where neither transmitter nor receiver are cognizant of the fading's realization, but both are aware of its statistic. Our focus is on the high signal-to-noise ratio (SNR) regime.

It has been shown in [1] for noncoherent frequency-flat fading channels that if the fading process is of finite entropy rate, then at high SNR capacity grows double-logarithmically with the SNR 1 For noncoherent multipath fading channels, it has been recently demonstrated that if the delay spread is large in the sense that the variances of the path gains do not decay faster than geometrically, then capacity is bounded in the SNR [3]. For such channels, capacity does not tend to infinity as the SNR tends to infinity.

The above condition can only be met by multipath fading channels that have an infinite number of paths in the sense that the channel output is influenced by the present and by all previous channel inputs. In this paper we consider multipath fading channels with a finite number of paths, i.e., the channel output is only influenced by the present and by the $L$ previous channel inputs. In order to characterize the capacity of this channel at high SNR, we study the capacity pre-loglog, defined as the limiting ratio of capacity to $\log \log$ SNR as SNR tends to infinity. We show that the pre-loglog is not diminished by the multipath behavior, i.e., irrespective of the value of $L$ the pre-loglog is 1 . To state this result precisely we begin with a mathematical description of the channel model.

\section{A. Channel Model}

Let $\mathbb{C}$ and $\mathbb{Z}^{+}$denote the set of complex numbers and the set of positive integers, respectively. We consider a discretetime multipath fading channel whose channel output $Y_{k} \in \mathbb{C}$

\footnotetext{
${ }^{1}$ It is well known that when the receiver knows the fading perfectly, then capacity increases logarithmically in the SNR [2]. Thus communicating over noncoherent flat-fading channels at high SNR is power inefficient.
}

at time $k \in \mathbb{Z}^{+}$corresponding to the time- 1 through time- $k$ channel inputs $x_{1}, \ldots, x_{k} \in \mathbb{C}$ is given by

$$
Y_{k}= \begin{cases}\sum_{\ell=0}^{k-1} H_{k}^{(\ell)} x_{k-\ell}+Z_{k}, & k=1, \ldots, \mathrm{L} \\ \sum_{\ell=0}^{\mathrm{L}} H_{k}^{(\ell)} x_{k-\ell}+Z_{k}, & k=\mathrm{L}+1, \mathrm{~L}+2, \ldots .\end{cases}
$$

Here $H_{k}^{(\ell)}$ denotes the time- $k$ gain of the $\ell$-th path; $\left\{Z_{k}\right\}$ is a sequence of independent and identically distributed (IID), zero-mean, variance- $\sigma^{2}$, circularly-symmetric, complex Gaussian random variables; and $L \in \mathbb{Z}_{0}^{+}$(where $\mathbb{Z}_{0}^{+}$denotes the set of nonnegative integers) denotes the number of paths that influence $Y_{k}$. For $\mathrm{L}=0$, the channel (1) reduces to the flat-fading channel that was studied in [1]; and for $L=\infty$, Equation (1) describes the multipath fading channel that was studied in [3]. In this paper we shall focus on the case where the number of paths is finite, i.e., where $L<\infty$. We assume that for each path $\ell=0, \ldots, \mathrm{L}$ the stochastic process $\left\{H_{k}^{(\ell)}, k \in \mathbb{Z}^{+}\right\}$is a zero-mean stationary process. We denote its variance and its differential entropy rate by

$$
\alpha_{\ell} \triangleq \mathrm{E}\left[\left|H_{k}^{(\ell)}\right|^{2}\right], \quad \ell=0 \ldots, \mathrm{L}
$$

and

$$
h_{\ell} \triangleq \lim _{n \rightarrow \infty} \frac{1}{n} h\left(H_{1}^{(\ell)}, \ldots, H_{n}^{(\ell)}\right), \quad \ell=0, \ldots, \mathrm{L},
$$

respectively. Without loss of generality, we assume that $\alpha_{0}>$ 0 . We further assume that

$$
\alpha_{\ell}<\infty, \quad \ell=0, \ldots, \mathrm{L}
$$

and

$$
\min _{\ell \in \mathcal{L}} h_{\ell}>-\infty
$$

where the set $\mathcal{L}$ is defined as $\mathcal{L} \triangleq\left\{\nu=0, \ldots, \mathrm{L}: \alpha_{\nu}>0\right\}$. We finally assume that the $\mathrm{L}+1$ processes

$$
\left\{H_{k}^{(0)}, k \in \mathbb{Z}^{+}\right\}, \ldots,\left\{H_{k}^{(\mathrm{L})}, k \in \mathbb{Z}^{+}\right\}
$$

are independent ("uncorrelated scattering"), that they are jointly independent of $\left\{Z_{k}\right\}$, and that the joint law of

$$
\left(\left\{Z_{k}\right\},\left\{H_{k}^{(0)}, k \in \mathbb{Z}^{+}\right\}, \ldots,\left\{H_{k}^{(\mathrm{L})}, k \in \mathbb{Z}^{+}\right\}\right)
$$

does not depend on the input sequence $\left\{x_{k}\right\}$. We consider a noncoherent channel model where neither the transmitter nor the receiver is cognizant of the realization of $\left\{H_{k}^{(\ell)}, k \in \mathbb{Z}^{+}\right\}$, $\ell=0, \ldots, \mathrm{L}$, but both are aware of their law. We do not assume that the path gains are Gaussian. 


\section{B. Channel Capacity}

Let $A_{m}^{n}$ denote the sequence $A_{m}, \ldots, A_{n}$. We define the capacity as

$$
C(\mathrm{SNR}) \triangleq \lim _{n \rightarrow \infty} \frac{1}{n} \sup I\left(X_{1}^{n} ; Y_{1}^{n}\right),
$$

where the supremum is over all joint distributions on $X_{1}, \ldots, X_{n}$ satisfying the power constraint

$$
\frac{1}{n} \sum_{k=1}^{n} \mathrm{E}\left[\left|X_{k}\right|^{2}\right] \leq \mathrm{P}
$$

and where SNR is defined as

$$
\mathrm{SNR} \triangleq \frac{\mathrm{P}}{\sigma^{2}} .
$$

It can be shown that for the above channel model

$$
\begin{aligned}
& \sup I\left(X_{1}^{m} ; Y_{1}^{m}\right)+\sup I\left(X_{1}^{n} ; Y_{1}^{n}\right) \\
& \quad \leq \sup I\left(X_{1}^{m+n} ; Y_{1}^{m+n}\right)+o(m+n), \quad m, n \in \mathbb{Z}^{+}
\end{aligned}
$$

(where $\lim _{(m+n) \rightarrow \infty} o(m+n) /(m+n)=0$ ) so that, by a trivial generalization of Fekete's lemm, 2 , the limit in (6) exists and is given by

$$
\lim _{n \rightarrow \infty} \frac{1}{n} \sup I\left(X_{1}^{n} ; Y_{1}^{n}\right)=\sup _{n \in \mathbb{Z}^{+}} \frac{1}{n} \sup I\left(X_{1}^{n} ; Y_{1}^{n}\right) .
$$

By Fano's inequality, no rate above $C(\mathrm{SNR})$ is achievable. (See [4] for a definition of an achievable rate.) We do not claim that there is a coding theorem associated with (6), i.e., that $C(\mathrm{SNR})$ is achievable. A coding theorem will, for example, hold if the processes

$$
\left\{H_{k}^{(0)}, k \in \mathbb{Z}^{+}\right\}, \ldots,\left\{H_{k}^{(\mathrm{L})}, k \in \mathbb{Z}^{+}\right\}
$$

are jointly ergodic, see [5, Thm. 2].

We define the capacity pre-loglog as

$$
\Lambda \triangleq \varlimsup_{\mathrm{SNR} \rightarrow \infty} \frac{C(\mathrm{SNR})}{\log \log \mathrm{SNR}} .
$$

For flat-fading channels (i.e., when $L=0$ ) we have $\Lambda=1$ [1]. For multipath fading channels with an infinite number of paths (i.e., when $L=\infty$ ), it has been shown in [3] that when the sequence $\left\{\alpha_{\ell}\right\}$ decays not faster than geometrically, then capacity is bounded in the SNR and hence $\Lambda=0$. One might therefore expect that the pre-loglog decays with L. It turns out, however, that this is not the case.

\section{Main Result}

Theorem 1: Consider the above channel model, and assume that $\mathrm{L}<\infty$. Then, irrespective of $\mathrm{L}$, the capacity pre-loglog is given by

$$
\Lambda=\lim _{\mathrm{SNR} \rightarrow \infty} \frac{C(\mathrm{SNR})}{\log \log \mathrm{SNR}}=1 .
$$

\section{Proof: See Section [I]}

Thus for finite L, the pre-loglog is not affected by the multipath behavior.

\footnotetext{
${ }^{2}$ Fekete's lemma states that if a sequence $\left\{a_{n}\right\}$ is superadditive, i.e., $a_{n}+$ $a_{m} \leq a_{m+n}, m, n \in \mathbb{Z}^{+}$, then the $\operatorname{limit}_{\lim _{n \rightarrow \infty}} a_{n} / n$ exists and is given by $\sup _{n \in \mathbb{Z}^{+}} a_{n} / n$.
}

\section{Proof of Theorem 1}

In Section $\amalg-A$ we derive a capacity upper bound and show that the ratio of this bound to $\log \log$ SNR tends to 1 as SNR tends to infinity. In Section $\amalg-B$ we propose a coding scheme which achieves a capacity pre-loglog of 1 . Both results combine to prove Theorem 1

\section{A. Converse}

We begin with the chain rule for mutual information [4]

$$
I\left(X_{1}^{n} ; Y_{1}^{n}\right)=\sum_{k=1}^{n} I\left(X_{1}^{n} ; Y_{k} \mid Y_{1}^{k-1}\right)
$$

and upper bound then each summand on the right-hand side (RHS) of (13) using the general upper bound for mutual information [1, Eq. (27)]

$$
\begin{aligned}
I\left(X_{1}^{n} ; Y_{k} \mid Y_{1}^{k-1}\right) \leq & \mathrm{E}\left[\log \left|Y_{k}\right|^{2}\right]-h\left(Y_{k} \mid X_{1}^{n}, Y_{1}^{k-1}\right) \\
& +\xi\left(1+\log \mathrm{E}\left[\left|Y_{k}\right|^{2}\right]-\mathrm{E}\left[\log \left|Y_{k}\right|^{2}\right]\right) \\
& +\log \Gamma(\xi)-\xi \log \xi+\log \pi
\end{aligned}
$$

for any $\xi>0$. Here $\Gamma(\cdot)$ denotes the Gamma function.

We evaluate the terms on the RHS of (14) individually. We use [3, Eq. (15)] to upper bound

$$
\mathrm{E}\left[\log \left|Y_{k}\right|^{2}\right] \leq \mathrm{E}\left[\log \left(\sigma^{2}+\sum_{\ell=0}^{k-1} \alpha_{\ell}\left|X_{k-\ell}\right|^{2}\right)\right]
$$

and [3, Eq. (24)] to lower bound

$$
\begin{aligned}
h\left(Y_{k} \mid X_{1}^{n}, Y_{1}^{k-1}\right) \geq & \mathrm{E}\left[\log \left(\sigma^{2}+\sum_{\ell=0}^{k-1} \alpha_{\ell}\left|X_{k-\ell}\right|^{2}\right)\right] \\
& +\inf _{\ell \in \mathcal{L}}\left(h_{\ell}-\alpha_{\ell}\right),
\end{aligned}
$$

where we define $\alpha_{\ell} \triangleq 0, \ell=\mathrm{L}+1, \mathrm{~L}+2, \ldots$. The next term is readily evaluated as

$$
\log \mathrm{E}\left[\left|Y_{k}\right|^{2}\right]=\log \left(\sigma^{2}+\sum_{\ell=0}^{k-1} \alpha_{\ell} \mathrm{E}\left[\left|X_{k-\ell}\right|^{2}\right]\right) .
$$

Finally, we use [3, Eq. (30)] to lower bound

$$
\begin{array}{r}
\mathrm{E}\left[\log \left|Y_{k}\right|^{2}\right] \geq \mathrm{E}\left[\log \left(\sigma^{2}+\sum_{\ell=0}^{k-1} \alpha_{\ell}\left|X_{k-\ell}\right|^{2}\right)\right]+\log \delta^{2} \\
-2 \epsilon(\delta, \eta)-\frac{2}{\eta}\left(\frac{2}{e}+\log (\pi e)\right)+\frac{2}{\eta} \inf _{\ell \in \mathcal{L}}\left(h_{\ell}-\alpha_{\ell}\right),
\end{array}
$$

where $0<\delta \leq 1,0<\eta<1$, and where $\epsilon(\delta, \eta)>0$ tends to zero as $\delta \downarrow 0$.

Subtracting (18) from (17), and lower bounding $\mathrm{E}\left[\log \left(\sigma^{2}+\sum_{\ell=0}^{k-1} \alpha_{\ell}\left|X_{k-\ell}\right|^{2}\right)\right] \geq \log \sigma^{2}$ yields

$$
\begin{aligned}
& \log \mathrm{E}\left[\left|Y_{k}\right|^{2}\right]-\mathrm{E}\left[\log \left|Y_{k}\right|^{2}\right] \\
& \quad \leq \log \left(1+\sum_{\ell=0}^{k-1} \alpha_{\ell} \mathrm{E}\left[\left|X_{k-\ell}\right|^{2}\right] / \sigma^{2}\right)+\Psi,
\end{aligned}
$$


where we define

$\Psi \triangleq \log \frac{1}{\delta^{2}}+2 \epsilon(\delta, \eta)+\frac{2}{\eta}\left(\frac{2}{e}+\log (\pi e)\right)-\frac{2}{\eta} \inf _{\ell \in \mathcal{L}}\left(h_{\ell}-\alpha_{\ell}\right)$.

Thus we obtain from (19), (16), (15), and (14)

$$
\begin{aligned}
& I\left(X_{1}^{n} ; Y_{k} \mid Y_{1}^{k-1}\right) \\
& \leq- \\
& \quad \inf _{\ell \in \mathcal{L}}\left(h_{\ell}-\alpha_{\ell}\right) \\
& \quad+\xi\left(1+\log \left(1+\sum_{\ell=0}^{k-1} \alpha_{\ell} \mathrm{E}\left[\left|X_{k-\ell}\right|^{2}\right] / \sigma^{2}\right)+\Psi\right) \\
& \quad+\log \Gamma(\xi)-\xi \log \xi+\log \pi .
\end{aligned}
$$

Let $\alpha^{(\mathrm{L})}$ be defined as

$$
\alpha^{(\mathrm{L})} \triangleq \sum_{\ell=0}^{\mathrm{L}} \alpha_{\ell}
$$

We choose now

$$
\xi=\left(1+\log \left(1+\alpha^{(\mathrm{L})} \mathrm{SNR}\right)\right)^{-1}
$$

and use that [1, Eq. (337)]

$$
\log \Gamma(\xi)=\log \frac{1}{\xi}+o(1)
$$

and that $\xi \log \xi=o(1)$ (where the term $o(1)$ vanishes as $\xi$ tends to zero) to obtain

$$
\begin{aligned}
& I\left(X_{1}^{n} ; Y_{k} \mid Y_{1}^{k-1}\right) \\
& \leq-\inf _{\ell \in \mathcal{L}}\left(h_{\ell}-\alpha_{\ell}\right) \\
& \quad+\frac{1+\log \left(1+\sum_{\ell=0}^{k-1} \alpha_{\ell} \mathrm{E}\left[\left|X_{k-\ell}\right|^{2}\right] / \sigma^{2}\right)+\Psi}{1+\log \left(1+\alpha^{(\mathrm{L})} \mathrm{SNR}\right)} \\
& \quad+\log \left(1+\log \left(1+\alpha^{(\mathrm{L})} \mathrm{SNR}\right)\right)+\log \pi+o(1) .
\end{aligned}
$$

Using (23) in (13), and noting that $\xi$-and hence also the correction term $o(1)$ - does not depend on $k$ yields then

$\frac{1}{n} I\left(X_{1}^{n} ; Y_{1}^{n}\right) \leq \log \left(1+\log \left(1+\alpha^{(\mathrm{L})} \mathrm{SNR}\right)\right)+\Upsilon_{n, \mathrm{P}}+o(1)$,

where we define $\Upsilon_{n, P}$ as

$$
\begin{aligned}
& \Upsilon_{n, \mathrm{P}} \triangleq \frac{1+\frac{1}{n} \sum_{k=1}^{n} \log \left(1+\sum_{\ell=0}^{k-1} \alpha_{\ell} \mathrm{E}\left[\left|X_{k-\ell}\right|^{2}\right] / \sigma^{2}\right)+\Psi}{1+\log \left(1+\alpha^{(\mathrm{L})} \mathrm{SNR}\right)} \\
&-\inf _{\ell \in \mathcal{L}}\left(h_{\ell}-\alpha_{\ell}\right)+\log \pi .
\end{aligned}
$$

Note that by Jensen's inequality

$$
\begin{aligned}
& \frac{1}{n} \sum_{k=1}^{n} \log \left(1+\sum_{\ell=0}^{k-1} \alpha_{\ell} \mathrm{E}\left[\left|X_{k-\ell}\right|^{2}\right] / \sigma^{2}\right) \\
& \quad \leq \log \left(1+\frac{1}{n} \sum_{k=1}^{n} \sum_{\ell=0}^{k-1} \alpha_{\ell} \mathrm{E}\left[\left|X_{k-\ell}\right|^{2}\right] / \sigma^{2}\right) \\
& \quad \leq \log \left(1+\alpha^{(\mathrm{L}) \mathrm{SNR})}\right.
\end{aligned}
$$

where the last inequality follows by rewriting the double sum as $\frac{1}{n} \sum_{k=1}^{n} \mathrm{E}\left[\left|X_{k}\right|^{2}\right] / \sigma^{2} \sum_{\ell=0}^{n-k} \alpha_{\ell}$, and by upper bounding then $\sum_{\ell=0}^{k-n} \alpha_{\ell} \leq \alpha^{(\mathrm{L})}$ and using the power constraint (7). Consequently, we can upper bound $\Upsilon_{n, \mathrm{P}}$ by

$$
\Upsilon_{n, \mathrm{P}} \leq 1+\Psi-\inf _{\ell \in \mathcal{L}}\left(h_{\ell}-\alpha_{\ell}\right)+\log \pi
$$

and obtain therefore from (6), (24), and (27)

$$
\begin{aligned}
C(\mathrm{SNR}) \leq & \log \left(1+\log \left(1+\alpha^{(\mathrm{L})} \mathrm{SNR}\right)\right)+1+\Psi \\
& -\inf _{\ell \in \mathcal{L}}\left(h_{\ell}-\alpha_{\ell}\right)+\log \pi+o(1) .
\end{aligned}
$$

Noting that $\xi \downarrow 0$ as SNR tends to infinity (and hence $\lim _{\mathrm{SNR} \rightarrow \infty} O(1)=0$ ), this yields the desired result

$$
\Lambda \triangleq \varlimsup_{\mathrm{SNR} \rightarrow \infty} \frac{C(\mathrm{SNR})}{\log \log \mathrm{SNR}} \leq 1 .
$$

\section{B. Direct Part}

In order to show that

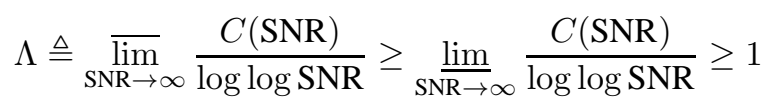

we shall derive a capacity lower bound and analyze then its ratio to $\log \log$ SNR as SNR tends to infinity.

To this end we evaluate $\frac{1}{n} I\left(X_{1}^{n} ; Y_{1}^{n}\right)$ for the following distribution on the inputs $\left\{X_{k}\right\}$. Let $\mathbf{X}_{b}=$ $\left(X_{b(\mathrm{~L}+\tau)+1}, \ldots, X_{(b+1)(\mathrm{L}+\tau)}\right)$ for some $\tau \in \mathbb{Z}^{+}$. We shall choose $\left\{\mathbf{X}_{b}\right\}$ to be IID with

$$
\mathbf{X}_{b}=(\underbrace{0, \ldots, 0}_{\mathrm{L}}, \tilde{X}_{b \tau+1}, \ldots, \tilde{X}_{(b+1) \tau}),
$$

where $\tilde{X}_{b \tau+1}, \ldots, \tilde{X}_{(b+1) \tau}$ is a sequence of independent, zero-mean, circularly-symmetric random variables with $\log \left|\tilde{X}_{b \tau+\nu}\right|^{2}$ being uniformly distributed over the interval $\left[\log x_{\min , \nu}^{2}, \log x_{\max , \nu}^{2}\right]$, i.e.,

$$
\log \left|\tilde{X}_{b \tau+\nu}\right|^{2} \sim \mathcal{U}\left(\left[\log x_{\min , \nu}^{2}, \log x_{\max , \nu}^{2}\right]\right), \quad \nu=1, \ldots, \tau .
$$

The parameters $x_{\min , \nu}$ and $x_{\max , \nu}$ will be chosen later.

Let $\kappa \triangleq\left\lfloor\frac{n}{L+\tau}\right\rfloor$, and let $\mathbf{Y}_{b}$ denote the vector $\left(Y_{b(\mathrm{~L}+\tau)+1}, \ldots, Y_{(b+1)(\mathrm{L}+\tau)}\right)$. We have

$$
\begin{aligned}
I\left(X_{1}^{n} ; Y_{1}^{n}\right) & \geq I\left(\mathbf{X}_{0}^{\kappa-1} ; \mathbf{Y}_{0}^{\kappa-1}\right) \\
& =\sum_{b=0}^{\kappa-1} I\left(\mathbf{X}_{b} ; \mathbf{Y}_{0}^{\kappa-1} \mid \mathbf{X}_{0}^{b-1}\right) \\
& \geq \sum_{b=0}^{\kappa-1} I\left(\mathbf{X}_{b} ; \mathbf{Y}_{b}\right)
\end{aligned}
$$

where the first inequality follows by restricting the number of observables in each of the terms; the subsequent equality follows by the chain rule for mutual information; and the last inequality follows by restricting the number of observables and by noting that $\left\{\mathbf{X}_{b}\right\}$ is IID.

We continue by lower bounding each summand on the RHS of (31) according to [6, Sec. III-B]. We use again the chain 
rule and that reducing observations cannot increase mutual information to obtain

$$
\begin{aligned}
I\left(\mathbf{X}_{b} ; \mathbf{Y}_{b}\right) & \geq I\left(\tilde{X}_{b \tau+1}^{(b+1) \tau} ; Y_{b(\mathrm{~L}+\tau)+\mathrm{L}+1}^{(b+1)(\mathrm{L}+\tau)}\right) \\
& =\sum_{\nu=1}^{\tau} I\left(\tilde{X}_{b \tau+\nu} ; Y_{b(\mathrm{~L}+\tau)+\mathrm{L}+1}^{(b+1)(\mathrm{L}+\tau)} \mid \tilde{X}_{b \tau+1}^{b \tau+\nu-1}\right) \\
& \geq \sum_{\nu=1}^{\tau} I\left(\tilde{X}_{b \tau+\nu} ; Y_{b(\mathrm{~L}+\tau)+\mathrm{L}+\nu}\right),
\end{aligned}
$$

where we additionally have used in the last inequality that $\tilde{X}_{b \tau+1}, \ldots, \tilde{X}_{(b+1) \tau}$ are independent.

Defining

$$
W_{b \tau+\nu} \triangleq \sum_{\ell=1}^{\mathrm{L}} H_{b(\mathrm{~L}+\tau)+\mathrm{L}+\nu}^{(\ell)} X_{b(\mathrm{~L}+\tau)+\mathrm{L}+\nu-\ell}+Z_{b(\mathrm{~L}+\tau)+\mathrm{L}+\nu}
$$

each summand on the RHS of (32) can be written as

$$
\begin{aligned}
& I\left(\tilde{X}_{b \tau+\nu} ; Y_{b(\mathrm{~L}+\tau)+\mathrm{L}+\nu}\right) \\
& \quad=I\left(\tilde{X}_{b \tau+\nu} ; H_{b(\mathrm{~L}+\tau)+\mathrm{L}+\nu}^{(0)} \tilde{X}_{b \tau+\nu}+W_{b \tau+\nu}\right) .
\end{aligned}
$$

A lower bound on (34) follows from the following lemma.

Lemma 2: Let the random variables $X, H$, and $W$ have finite second moments. Assume that both $X$ and $H$ are of finite differential entropy. Finally, assume that $X$ is independent of $H$; that $X$ is independent of $W$; and that $X-H-W$ forms a Markov chain. Then,

$$
\begin{aligned}
I(X ; H X+W) \geq & h(X)-\mathrm{E}\left[\log |X|^{2}\right]+\mathrm{E}\left[\log |H|^{2}\right] \\
& -\mathrm{E}\left[\log \left(\pi e\left(\sigma_{H}+\frac{\sigma_{W}}{|X|}\right)^{2}\right)\right]
\end{aligned}
$$

where $\sigma_{W}^{2} \geq 0$ and $\sigma_{H}^{2}>0$ are the variances of $W$ and $H$, respectively 3

Proof: See [6, Lemma 4].

It can be easily verified that for the channel model given in Section $[-\mathrm{A}$ and for the above coding scheme the lemma's conditions are satisfied. We can therefore lower bound $I\left(\tilde{X}_{b \tau+\nu} ; Y_{b(\mathrm{~L}+\tau)+\mathrm{L}+\nu}\right)$ by

$$
\begin{aligned}
& I\left(\tilde{X}_{b \tau+\nu} ; Y_{b(\mathrm{~L}+\tau)+\mathrm{L}+\nu}\right) \\
& \geq h\left(\tilde{X}_{b \tau+\nu}\right)-\mathrm{E}\left[\log \left|\tilde{X}_{b \tau+\nu}\right|^{2}\right]+\mathrm{E}\left[\log \left|H_{b(\mathrm{~L}+\tau)+\mathrm{L}+\nu}^{(0)}\right|^{2}\right] \\
& \quad-\mathrm{E}\left[\log \left(\pi e\left(\sqrt{\alpha_{0}}+\frac{\sqrt{\mathrm{E}\left[\left|W_{b \tau+\nu}\right|^{2}\right]}}{\left|\tilde{X}_{b \tau+\nu}\right|}\right)^{2}\right)\right]
\end{aligned}
$$

Using that the differential entropy of a circularly-symmetric random variable is given by (e.g., [1, Eqs. (320) \& (316)])

$$
h\left(\tilde{X}_{b \tau+\nu}\right)=\mathrm{E}\left[\log \left|\tilde{X}_{b \tau+\nu}\right|^{2}\right]+h\left(\log \left|\tilde{X}_{b \tau+\nu}\right|^{2}\right)+\log \pi,
$$

\footnotetext{
${ }^{3}$ Note that the assumptions that $X$ and $H$ have finite second moments and are of finite differential entropy guarantee that $\mathrm{E}\left[\log |X|^{2}\right]$ and $\mathrm{E}\left[\log |H|^{2}\right]$ are finite, see [1. Lemma 6.7e)].
}

and evaluating $h\left(\log \left|\tilde{X}_{b \tau+\nu}\right|^{2}\right)$ for our choice of $\tilde{X}_{b \tau+\nu}$, we obtain for the first two terms on the RHS of (36)

$h\left(\log \left|\tilde{X}_{b \tau+\nu}\right|^{2}\right)-\mathrm{E}\left[\log \left|\tilde{X}_{b \tau+\nu}\right|^{2}\right]=\log \log \frac{x_{\max , \nu}^{2}}{x_{\min , \nu}^{2}}+\log \pi$.

Upper bounding

$$
\begin{aligned}
\mathrm{E}\left[\left|W_{b \tau+\nu}\right|^{2}\right] & =\sum_{\ell=1}^{\mathrm{L}} \alpha_{\ell} \mathrm{E}\left[\left|X_{b(\mathrm{~L}+\tau)+\mathrm{L}+\nu-\ell}\right|^{2}\right]+\sigma^{2} \\
& \leq \max _{\ell=0, \ldots, \nu-1} x_{\max , \ell}^{2} \cdot \alpha^{(\mathrm{L})}+\sigma^{2}
\end{aligned}
$$

(where we define $x_{\max , 0}^{2} \triangleq 0$ ), and lower bounding $\left|\tilde{X}_{b \tau+\nu}\right|^{2} \geq$ $x_{\min , \nu}^{2}$, the last term on the RHS of (36) can be upper bounded by

$$
\begin{aligned}
& \mathrm{E}\left[\log \left(\pi e\left(\sqrt{\alpha_{0}}+\frac{\sqrt{\mathrm{E}\left[\left|W_{b \tau+\nu}\right|^{2}\right]}}{\left|\tilde{X}_{b \tau+\nu}\right|}\right)^{2}\right)\right] \\
& \leq \log \left(\pi e\left(\sqrt{\alpha_{0}}+\sqrt{\frac{\max _{\ell=0, \ldots, \nu-1} x_{\max , \ell}^{2} \cdot \alpha^{(\mathrm{L})}+\sigma^{2}}{x_{\min , \nu}^{2}}}\right)^{2}\right)
\end{aligned}
$$

and we thus obtain from (36), (38), and (40)

$$
\begin{aligned}
& I\left(\tilde{X}_{b \tau+\nu} ; Y_{b(\mathrm{~L}+\tau)+\mathrm{L}+\nu}\right) \\
& \geq \log \log \frac{x_{\max , \nu}^{2}}{x_{\min , \nu}^{2}}+\mathrm{E}\left[\log \left|H_{b(\mathrm{~L}+\tau)+\mathrm{L}+\nu}^{(0)}\right|^{2}\right]-1 \\
& \quad-2 \log \left(\sqrt{\alpha_{0}}+\sqrt{\frac{\max _{\ell=0, \ldots, \nu-1} x_{\max , \ell}^{2} \cdot \alpha^{(\mathrm{L})}+\sigma^{2}}{x_{\min , \nu}^{2}}}\right) .
\end{aligned}
$$

Following [6, Eqs. (102) \& (103)], we choose now (assuming that $P>1$ )

$$
\begin{array}{ll}
x_{\max , \nu}^{2}=\mathrm{P}^{\nu / \tau}, & \nu=1, \ldots, \tau \\
x_{\min , \nu}^{2}=\mathrm{P}^{(\nu-1) / \tau} \log \mathrm{P}, & \nu=1, \ldots, \tau .
\end{array}
$$

With this choice we have

$$
\frac{x_{\max , \nu}^{2}}{x_{\min , \nu}^{2}}=\frac{\mathrm{P}^{1 / \tau}}{\log \mathrm{P}}, \quad \nu=1, \ldots, \tau
$$

and

$$
\frac{\max _{\ell=0, \ldots, \nu-1} x_{\max , \ell}^{2}}{x_{\min , \nu}^{2}}= \begin{cases}0, & \nu=1 \\ 1 / \log \mathrm{P}, & \nu=2, \ldots, \tau .\end{cases}
$$

Thus applying (42) \& (43) to (41) yields

$$
\begin{aligned}
& I\left(\tilde{X}_{b \tau+\nu} ; Y_{b(\mathrm{~L}+\tau)+\mathrm{L}+\nu}\right) \\
& \geq \log \log \frac{\mathrm{P}^{1 / \tau}}{\log \mathrm{P}}+\mathrm{E}\left[\log \left|H_{b(\mathrm{~L}+\tau)+\mathrm{L}+\nu}^{(0)}\right|^{2}\right]-1 \\
& \quad-2 \log \left(\sqrt{\alpha_{0}}+\sqrt{\left.\frac{\alpha^{(\mathrm{L})}}{\log \mathrm{P}}+\frac{\sigma^{2}}{\mathrm{P}^{(\nu-1) / \tau} \log \mathrm{P}}\right)}\right. \\
& \geq \log \log \frac{\mathrm{P}^{1 / \tau}}{\log \mathrm{P}}+\mathrm{E}\left[\log \left|H_{1}^{(0)}\right|^{2}\right]-1
\end{aligned}
$$




$$
-2 \log \left(\sqrt{\alpha_{0}}+\sqrt{\frac{\alpha^{(\mathrm{L})}+\sigma^{2}}{\log \mathrm{P}}}\right), \quad \mathrm{P}>1,
$$

where the last inequality follows by using that the process $\left\{H_{k}^{(0)}, k \in \mathbb{Z}^{+}\right\}$is stationary and that, for $\mathrm{P}>1$, $\mathrm{P}^{(\nu-1) / \tau} \geq 1$.

Note that the RHS of (44) depends neither on $\nu$ nor on $b$. We therefore obtain from (44), (32), and (31)

$$
I\left(X_{1}^{n} ; Y_{1}^{n}\right) \geq \kappa \tau \log \log \frac{\mathrm{P}^{1 / \tau}}{\log \mathrm{P}}+\kappa \tau \Xi_{\mathrm{P}}, \quad \mathrm{P}>1,
$$

where we define $\Xi_{\mathrm{p}}$ as

$$
\Xi_{\mathrm{P}} \triangleq \mathrm{E}\left[\log \left|H_{1}^{(0)}\right|^{2}\right]-1-2 \log \left(\sqrt{\alpha_{0}}+\sqrt{\frac{\alpha^{(\mathrm{L})}+\sigma^{2}}{\log \mathrm{P}}}\right) .
$$

Dividing the RHS of (45) by $n$, and computing the limit as $n$ tends to infinity yields the capacity lower bound

$$
\begin{aligned}
C(\mathrm{SNR}) & \geq \varliminf_{n \rightarrow \infty} \frac{1}{n} I\left(X_{1}^{n} ; Y_{1}^{n}\right) \\
& \geq \frac{\tau}{\mathrm{L}+\tau} \log \log \frac{\mathrm{P}^{1 / \tau}}{\log \mathrm{P}}+\frac{\tau}{\mathrm{L}+\tau} \Xi_{\mathrm{P}}, \quad \mathrm{P}>1,
\end{aligned}
$$

where we have used that $\lim _{n \rightarrow \infty} \kappa / n=1 /(\mathrm{L}+\tau)$.

By noting that (for any fixed $\tau$ )

$$
\begin{aligned}
\lim _{\mathrm{SNR} \rightarrow \infty} \frac{\log \log \left(\mathrm{P}^{1 / \tau} / \log \mathrm{P}\right)}{\log \log \mathrm{SNR}} & =1 \\
\lim _{\mathrm{SNR} \rightarrow \infty} \frac{\Xi_{\mathrm{P}}}{\log \log \mathrm{SNR}} & =0
\end{aligned}
$$

we infer from (47) that the capacity pre- $\log \log \Lambda$ is lower bounded by

$$
\Lambda \triangleq \varlimsup_{\mathrm{SNR} \rightarrow \infty} \frac{C(\mathrm{SNR})}{\log \log \mathrm{SNR}} \geq \underset{\mathrm{SNR} \rightarrow \infty}{\lim _{\log \log \mathrm{SNR}}} \geq \frac{C(\mathrm{SNR})}{\mathrm{L}+\tau}
$$

The claim (30) follows now by letting $\tau$ tend to infinity.

\section{CONCLUSION}

We considered a discrete-time, noncoherent, multipath fading channel where the number of paths is finite, i.e., where the channel output is influenced by the present and by the $\mathrm{L}$ previous channel inputs. It was shown that, irrespective of the number of paths, the capacity pre-loglog is 1 (which is equal to the pre-loglog of a flat-fading channel). Thus, when the number of paths is finite, then the multipath behavior has no significant effect on the high-SNR capacity. This is perhaps surprising as it has been shown in [3] that if the channel output is influenced by the present and by all previous channel inputs, and if the variances of the path gains do not decay faster than geometrically, then capacity is bounded in the SNR. For such channels the capacity does not tend to infinity as the SNR tends to infinity and hence the capacity pre-loglog is zero.

The above results indicate that the high-SNR behavior of the capacity of noncoherent multipath fading channels depends critically on the assumed channel model. Thus, when studying such channels at high SNR, one has to attach great importance to the channel modeling, as slight changes in the model might lead to completely different capacity results.

\section{ACKNOWLEDGMENT}

Fruitful discussions with Helmut Bölcskei and Giuseppe Durisi are gratefully acknowledged.

\section{REFERENCES}

[1] A. Lapidoth and S. M. Moser, "Capacity bounds via duality with applications to multiple-antenna systems on flat fading channels," IEEE Trans. Inform. Theory, vol. 49, no. 10, pp. 2426-2467, Oct. 2003.

[2] T. H. E. Ericson, "A Gaussian channel with slow fading," IEEE Trans. Inform. Theory, vol. 16, no. 3, pp. 353-355, May 1970.

[3] T. Koch and A. Lapidoth, "Multipath channels of bounded capacity," in Proc. Inform. Theory Workshop (ITW), Porto, Portugal, May 5-9, 2008.

[4] T. M. Cover and J. A. Thomas, Elements of Information Theory. John Wiley \& Sons, 1991.

[5] Y.-H. Kim, "A coding theorem for a class of stationary channels with feedback," IEEE Trans. Inform. Theory, vol. 54, no. 4, pp. 1488-1499, Apr. 2008.

[6] A. Lapidoth, "On the high-SNR capacity of noncoherent networks," IEEE Trans. Inform. Theory, vol. 51, no. 9, pp. 3025-3036, Sept. 2005. 